\title{
Die Unterwasserreinigung von Seeschiffen als Herausforderung für das Gewässerschutzrecht
}

\author{
Valentin J. Schatz*
}

(c) Der/die Autor(en) 2021. Dieser Artikel ist eine Open-Access-Publikation.

Dieser Beitrag knüpft thematisch an den in NuR 2021, 361 ff. erschienenen Beitrag „Die Regulierung der Einführung invasiver gebietsfremder Arten durch Biofouling an der Schnittstelle zwischen Naturschutzrecht und Seeschifffahrtsrecht" an. Er widmet sich speziell den rechtlichen Rahmenbedingungen der Unterwasserreinigung von Seeschiffen als einer Maßnahme des Biofouling Managements, die voraussichtlich auch in Deutschland an Bedeutung gewinnen wird. Der Beitrag untersucht die Zulässigkeit der Unterwasserreinigung aus Sicht des Gewässerschutzrechts im weiteren Sinne und zeigt, dass hierfür nicht das umweltbezogene (Binnen-) oder Seeschifffahrtsrecht maßgeblich ist. Nach dem stattdessen ausschlaggebenden Wasserhaushalts- und Meeresumweltrecht hängt die Zulässigkeit der Unterwasserreinigung von Seeschiffen davon $a b$, ob bzw. wie gut die angewandte Methode bzw. Technologie in der Lage ist, die dabei freigesetzten Stoffe aufzufangen und einer Verwertung oder Entsorgung an Land im Einklang mit dem Kreislaufwirtschaftsrecht zuzuführen.

\section{Einleitung}

Der Bewuchs der unter Wasser befindlichen Oberflächen von Seeschiffen durch Mikroorganismen und Makroorganismen (sog. Biofouling) ${ }^{1}$ bringt eine Reihe von Nachteilen mit sich, die sowohl ökologischer Natur (insb. Einführung gebietsfremder invasiver Arten) als auch ökonomischer Natur (insb. Erhöhung des Treibstoffverbrauchs der Schiffe) sind und die durch sog. Biofouling Management adressiert werden sollen. Die rechtliche Regulierung des Biofouling Managements im Hinblick auf die Einführung gebietsfremder invasiver Arten war bereits Gegenstand eines früheren Beitrags. ${ }^{2}$ Daran anknüpfend widmet sich dieser Beitrag speziell den rechtlichen Rahmenbedingungen der Unterwasserreinigung von Seeschiffen (sog. in-water cleaning oder underwater cleaning) als einer international und auch in Europa zunehmend an Bedeutung gewinnenden Maßnahme des Biofouling Managements, die künftig auch in Deutschland zum Einsatz kommen dürfte. Da die Möglichkeit, eine Unterwasserreinigung durchzuführen von den Reedereien, deren Schiffe deutsche Häfen anfahren, zunehmend nachgefragt werden dürfte und in Deutschland bereits potenzielle private Anbieter von Unterwasserreinigungen existieren, ist die möglichst baldige Herausbildung einer einheitlichen deutschen Rechtspraxis erstrebenswert. ${ }^{3}$

Der Beitrag stellt zunächst kurz dar, inwieweit die Unterwasserreinigung in einem Spannungsverhältnis zum Gewässerschutz steht. Danach geht er auf die Regulierung des Biofouling Managements durch die Internationale Seeschifffahrtsorganisation (IMO) ein, soweit diese die Unterwasserreinigung als Maßnahme des Biofouling Manage-

\section{Valentin J. Schatz,}

Referendar am Hanseatischen Oberlandesgericht,

Wissenschaftlicher Mitarbeiter am Institut für Seerecht und Seehandelsrecht, Universität Hamburg,

Hamburg, Deutschland ments ins Auge fasst. Den Kern des Beitrags bildet eine Untersuchung des deutschen Rechts, wobei detailliert auf die in den unterschiedlichen Gewässertypen einschlägigen Regelungen eingegangen wird. Die Ergebnisse der Untersuchung werden in einer Schlussbetrachtung zusammengefasst und eingeordnet.

\section{Unterwasserreinigung im ökologischen Spannungsfeld des Biofouling Managements}

Wie bereits an anderer Stelle erläutert, kann Biofouling ein Vektor beim Transport von invasiven gebietsfremden Arten in aquatische Ökosysteme sein. ${ }^{4}$ Zusätzlich erhöht Biofouling insbesondere auch den Reibungswiderstand von Schiffen, was wiederum zu einer reduzierten Geschwindigkeit und einem erhöhtem Treibstoffbedarf - und damit wiederum zu erhöhten Emissionen - führt. ${ }^{5}$ Umgekehrt können aus Emissionsgründen angestrebte langsamere Geschwindigkeiten von Seeschiffen den Bewuchs durch Organismen beschleunigen, da es den Organismen so leichter fallen kann, sich auf Oberflächen anzusiedeln. ${ }^{6}$ In Anbetracht

* Dieser Aufsatz entstand während einer Verwaltungsstation beim Bundesamt für Seeschifffahrt und Hydrographie (BSH). Er gibt allein die Auffassung des Autors wieder. Besonderer Dank für wertvolle Anmerkungen gilt Dipl.-Ing. Donna-Lee Garrick (Referat Qualitative Wasserwirtschaft, SKUMS Bremen), Dr. Susanne Heitmüller (BSH) und Hannelore Keim (Bundesministerium für Verkehr und digitale Infrastruktur (BMVI)).

1) Umweltbundesamt (UBA): Bewuchsatlas: Was ist Bewuchs? Stand 14.3.2021, abrufbar unter https://www.umweltbundesamt.de/bewuchsatlas-was-ist-bewuchs; Bundesamt für Seeschifffahrt und Hydrographie (BSH): Biofouling, Stand 14.3. 2021, abrufbar unter: https://www.bsh.de/DE/THEMEN/ Schifffahrt/Umwelt_und_Schifffahrt/Biofouling/biofouling node.html;jsessionid=255D5C629E3519C445D7F5F8EE8F B9C3.live11291.

2) Schatz, Die Regulierung der Einführung invasiver gebietsfremder Arten durch Biofouling an der Schnittstelle zwischen Naturschutzrecht und Seeschifffahrtsrecht, NuR 2021, $361 \mathrm{ff}$

3) Informationen über potenzielle Anbieter einer Unterwasserreinigung in deutschen Häfen stellt künftig das BSH zur Verfügung. Siehe BIOFOULING Management Database des BSH, Stand 14.3.2021, abrufbar unter https://biofouling-database.bsh.de/. Hierbei handelt es sich um einen Informationsservice, nicht aber um eine inhaltliche Positionierung des BSH zur Zulässigkeit der Unterwasserreinigung.

4) Siehe zu Biofouling Management im Hinblick auf die Einführung invasiver gebietsfremder Arten Schatz, Die Regulierung der Einführung invasiver gebietsfremder Arten durch Biofouling an der Schnittstelle zwischen Naturschutzrecht und Seeschifffahrtsrecht, NuR 2021, $361 \mathrm{ff}$

5) Siehe allgemein zu Biofouling Management und Schifffahrt: Davidson/Scianni/Hewitt/Everett/Holm/Tamburri/Ruiz, Mini-Review: Assessing the Drivers of Ship Biofouling Management: Aligning Industry and Biosecurity Goals, Biofouling 2016, 411.

6) Edyvean, Consequences of Fouling on Shipping, in: Dürr/Thomason (Hrsg.), Biofouling, 2010, S. 217. 
dieser Wechselwirkungen ist Biofouling ein auch klimapolitisch bedeutsames Thema. ${ }^{7}$

Um Biofouling vorzubeugen, verfügen Schiffe üblicherweise über Antifouling-Beschichtungen (anti-fouling systems (AFS)). ${ }^{8}$ Traditionelle AFS enthalten üblicherweise umweltschädliche Biozide, die im Laufe der Zeit im Wasser freigesetzt werden. ${ }^{9}$ Der Einsatz von AFS mit Bioziden ist mittlerweile aber stark reguliert (etwa durch das Internationale Übereinkommen über die Beschränkung des Einsatzes schädlicher Bewuchsschutzsysteme auf Schiffen von 2001 $(\text { AFS-Übereinkommen) })^{10}$ und teilweise verboten (das gilt beispielsweise für Tributylzinn (TBT)). ${ }^{11}$ Daher dürfte alternativen AFS sowie regelmäßigen Reinigungsverfahren für Schiffe künftig große Bedeutung zukommen.

Soweit ersichtlich kam eine Reinigung von Schiffen bisher vor allem im Trockendock in Betracht, wobei allerdings bei regulären Wartungen regelmäßig das AFS vollständig ausgetauscht und nicht lediglich gereinigt wurde. Eine Alternative dazu ist die Unterwasserreinigung im Hafen oder auf Reede, bei der der Bewuchs direkt im Wasser entfernt wird. Dies kann entweder mechanisch oder mit Wasserhochdruck (also ohne Einsatz von Chemikalien) durch Taucher oder ferngesteuerte Unterwasserfahrzeuge (remotely operated underwater vehicles (ROVs)) erfolgen. ${ }^{12}$ Bei diesen Reinigungsarbeiten fällt Biomasse an, die - wenn lebensfähig - zu einer Einführung invasiver gebietsfremder Arten führen kann. ${ }^{13}$ Insbesondere beim Entfernen von Makroorganismen werden darüber hinaus häufig auch (biozide) Beschichtungen und Farbresten freigesetzt. ${ }^{14}$

Obwohl die Unterwasserreinigung letztlich zur Erhaltung mariner Ökosysteme beitragen soll, ${ }^{15}$ kann sie folglich in einem Spannungsverhältnis zum Gewässerschutz stehen, wenn die freigesetzten Stoffe nicht sachgerecht aufgefangen und entsorgt werden. Hier zeigt sich eine Parallele zur Problematik des Ballastwassers. Nach dem Internationalen Übereinkommen zur Kontrolle und Behandlung von Ballastwasser und Sedimenten von Schiffen von 2004 ist im Rahmen der Bekämpfung der Einführung invasiver gebietsfremder Arten der Einsatz von chemischen und physikalischen Methoden zur Behandlung von Ballastwasser zulässig, was wiederum die Frage nach dem richtigen Umgang mit dem so behandelten Ballastwasser aufwirft. ${ }^{16}$

Es stellt sich folglich die Frage, unter welchen technischen Voraussetzungen die Unterwasserreinigung von Seeschiffen erlaubt bzw. erlaubnisfähig ist. Diesbezüglich ist vor allem im Hinblick auf die Entsorgung der entfernten Biomasse (samt möglichen Resten von bioziden Beschichtungen und Farben) zu klären, ob diese im Wasser belassen werden kann oder ob sie aufgefangen und an Land entsorgt werden muss.

\section{Globale Standards}

\subsection{Seevölkerrechtlicher Rahmen}

Auf der globalen Ebene existieren bisher keine speziellen, rechtsverbindlichen Regelungen für das Biofouling $\mathrm{Ma}$ nagement oder gar die Unterwasserreinigung. ${ }^{17}$ Allerdings enthält das Seerechtsübereinkommen der Vereinten Nationen (SRÜ) von 1982 allgemeine seevölkerrechtliche Verpflichtungen sowohl im Hinblick auf die Einführung gebietsfremder invasiver Arten ${ }^{18}$ als auch auf den Schutz von Meeresgewässern vor Verschmutzung. ${ }^{19}$ Das Einbringen von Stoffen in das Meer, wie im Rahmen einer Unterwasserreinigung der Fall sein kann, ist zudem unter anderem Gegenstand des Übereinkommens über die Verhütung der Meeresverschmutzung durch das Einbringen von Abfällen und anderen Stoffen (Londoner Übereinkommen) von 1972 und des Protokolls zum Übereinkommen über die Verhütung der Meeresverschmutzung durch das Einbringen von Abfällen und anderen Stoffen (Londoner Protokoll) von 1996, auf dessen national-rechtliche Umsetzung in Deutschland unten noch einzugehen sein wird. Insgesamt kann der seevölkerrechtliche Rahmen für das Biofouling Management und insbesondere die Unterwasserreinigung als rudimentär und im Rahmen internationaler und nationaler Regelungen ausgestaltungsbedürftig bezeichnet werden.

\subsection{IMO Biofouling-Richtlinien: Unterwasserreinigung als Maßnahme des Biofouling Managements}

Trotz des Fehlens spezieller seevölkerrechtlicher Regelungen wurde das Thema Biofouling Management bereits im Rahmen der IMO thematisiert, die mit den IMO Biofouling-Richtlinien von 2011 rechtlich nicht verbindliche

7) Poloczanska/Butler, Biofouling and Climate Change, in: Dürr/ Thomason (Hrsg.), Biofouling, 2010, S. 333. Umgekehrt wirken sich ozeanische Einflüsse des Klimawandels wiederum auf Biofouling aus. Siehe dazu etwa Dobretsov/Coutinho/Rittschof/Salta/ Ragazzola/Hellio, The Oceans Are Changing: Impact of Ocean Warming and Acidification on Biofouling Communities, Biofouling 2019, 585.

8) Umweltbundesamt (UBA): Antifouling-Mittel, Stand 14.3.2021, abrufbar unter: https://www.umweltbundesamt.de/themen/ chemikalien/biozide/biozidprodukte/antifouling-mittel.

9) Thomas/Brooks, The Environmental Fate and Effects of Antifouling Paint Biocides, Biofouling 2010, 73; Hallers-Tjabbes/Walmsley, Consequences of Antifouling Systems: An Environmental Perspective, in: Dürr/Thomason (Hrsg.), Biofouling, 2010, S. 243.

10) Siehe auch Verordnung (EU) Nr. 528/2012 des Europäischen Parlaments und des Rates v. 22.5.2012 über die Bereitstellung auf dem Markt und die Verwendung von Biozidprodukten, ABl. 2012 L 167, S. 1, Produktart 21 („Antifouling-Produkte“).

11) Siehe dazu Cheyne, Regulation of Marine Antifouling in International and EC Law, in: Dürr/Thomason (Hrsg.), Biofouling, 2010, S. 306; Cheyne, Implications of International and European Regulatory Developments for Marine Antifouling, in: Dürr/Thomason (Hrsg.), Biofouling, 2010, S. 388; Kymenvaara/ Tegnér Anker/Baaner/Ekroos/Gipperth/Seppälä, Regulating Antifouling Paints for Leisure Boats: A Patchwork of Rules Across Three Baltic Sea Countries, Nordic Environmental Law Journal 2017, 7; Harrison, Saving the Oceans Through Law: The International Legal Framework for the Protection of the Marine Environment, 2017, S. $130 \mathrm{ff}$.

12) Siehe z.B. LIMNOMAR, Projekt „CLEAN“: Unterwasserreinigung in der professionellen Schifffahrt, Stand: 14.3.2021, abrufbar unter: http://www.limnomar.de/index.php?\&modul=gruppe\& grp=2\&pid=46; CleanSubSea, Envirocart: The Worlds Most Advanced In-water Hull Cleaning System, Stand: 14.3.2021, abrufbar unter https://www.cleansubsea.com.au/technology/.

13) C. Woods/Floerl/Jones, Biosecurity Risks Associated with In-water and Shore-based Marine Vessel Hull Cleaning Operations, Marine Pollution Bulletin 2012, 1392.

14) Watermann/Herlyn, Beschichtungspartikel- und Polymereinträge aus Unterwasserbeschichtungen von Schiffen und Booten, Wasser und Abfall 2020, 43; Oliveira/Granhag, Ship Hull In-water Cleaning and its Effects on Fouling-control Coatings, Biofouling $2020,332$.

15) Vgl. dazu Ziel 14 der UN, Transforming Our World: The 2030 Agenda for Sustainable Development (25.9.2015), UN. Doc. A/ $\mathrm{RES} / 70 / 1$.

16) Vgl. Proelß/Schatz, Rechtliche Vorgaben zum Umgang mit Schiffsabwasser: Völker-, unions- und nationalrechtliche Anforderungen an Einleitungen von Scrubber-Abwasser, Ballastwasser und häuslichem Abwasser durch Schiffe, 2019, S. $92 \mathrm{ff}$.

17) Schatz, Die Regulierung der Einführung invasiver gebietsfremder Arten durch Biofouling an der Schnittstelle zwischen Naturschutzrecht und Seeschifffahrtsrecht, NuR 2021, $361 \mathrm{ff}$.

18) Art. 196 SRÜ. Siehe dazu Schatz, Die Regulierung der Einführung invasiver gebietsfremder Arten durch Biofouling an der Schnittstelle zwischen Naturschutzrecht und Seeschifffahrtsrecht, NuR 2021, $361 \mathrm{ff}$.

19) Art. 192 ff. SRÜ. Siehe dazu etwa Harrison, Saving the Oceans Through Law: The International Legal Framework for the Protection of the Marine Environment, 2017, S. $17 \mathrm{ff}$., $92 \mathrm{ff}$. 
Empfehlungen geschaffen hat. ${ }^{20}$ Diese Richtlinien enthalten praktische Empfehlungen für Maßnahmen, die dazu beitragen sollen, Risiken der Verbreitung von nicht-einheimischen Arten durch Biofouling sowie ökologische Risiken von Biofouling Management zu minimieren. ${ }^{21}$

Die Unterwasserinspektion, -reinigung und -wartung wird im siebten Abschnitt der Biofouling-Richtlinien thematisiert. Dort wird darauf hingewiesen, dass sich auch während der vorgesehenen Lebensdauer des AFS unerwünschte Mengen an Biofouling ansammeln können. ${ }^{22}$ Es könne daher ratsam sein, Inspektionen, Reinigungen und Wartungen von Schiffen durchzuführen, um es frei von Bewuchs zu halten. ${ }^{23}$ Vor diesem Hintergrund könne die Unterwasserreinigung ein wichtiger Teil des Biofouling Managements sein. ${ }^{24}$ Im Vergleich zum Makrobewuchs könne der Mikrobewuchs mit schonenderen Techniken entfernt werden, die den Abrieb des AFS und/oder die Biozidfreisetzung minimieren. ${ }^{25}$ Die Entfernung von Mikrobewuchs könne den Wirkungsgrad des Schiffsrumpfes verbessern und damit den Treibstoffverbrauch und die Treibhausgasemissionen reduzieren. ${ }^{26}$ Vor diesem Hintergrund solle der Schiffsrumpf nach Möglichkeit mit sanften Methoden gereinigt werden, wenn signifikantes Microfouling auftrete. ${ }^{27}$ Die zeitige Reinigung könne auch das Risiko der Ausbreitung invasiver aquatischer Arten verringern, indem die Ansammlung von Makrobewuchs verhindert werde. ${ }^{28}$

Allerdings weisen die Richtlinien zugleich darauf hin, dass die Unterwasserreinigung Umweltrisiken mit sich bringen könne, die jeweils von der Art des Biofoulings (Mikrobewuchs oder Makrobewuchs), der Menge der freigesetzten Rückstände des AFS und dem bioziden Gehalt des AFS abhängen könnten. ${ }^{29}$ Aus diesem Grund könne es für Staaten angebracht sein, eine Risikobeurteilung durchzuführen, um das Risiko einer Unterwasserreinigung zu bewerten und potenzielle Gefahren für die Umwelt zu minimieren. ${ }^{30}$

Die Biofouling-Richtlinien listen eine Reihe von Faktoren auf, die Gegenstand einer solchen Risikobeurteilung sein könnten. ${ }^{31}$ Konkret seien dies das biologische Risiko des Entfernens der Biofouling-Organismen vom Schiff (einschließlich der Lebensfähigkeit der Biofouling-Organismen und der Fähigkeit, freigesetzte Biomasse aufzufangen), ${ }^{32}$ Faktoren, die die Entstehung von Biofouling beeinflussen können, wie etwa Änderungen des Betriebsprofils des Schiffes, ${ }^{33}$ das geografische Gebiet, aus dem das Biofouling auf dem Schiff stammt (sofern bekannt), ${ }^{34}$ und toxische Wirkungen im Zusammenhang mit Stoffen innerhalb des AFS, die während der Reinigungstätigkeit freigesetzt werden könnten und jede nachfolgende Beschädigung des AFS. ${ }^{35}$

Nach Ziff. 7.7 S. 1 der Biofouling-Richtlinien soll Personal, das vorschlägt, eine Unterwasserreinigung durchzuführen, alle Vorschriften oder Anforderungen für die Durchführung einer Unterwasserreinigung kennen, einschließlich aller Vorschriften über die Einleitung von Chemikalien in die Meeresumwelt und die Lage empfindlicher Gebiete (z.B. Meeresschutzgebiete und Ballastwasseraustauschgebiete). Wo ein erhebliches Wachstum von Makrobewuchs festgestellt werde, soll das Biofouling entfernt oder (wenn dies ohne Beschädigung des AFS möglich ist) gemäß den Biofouling-Richtlinien behandelt werden. ${ }^{36}$ Wo verfügbar, soll eine geeignete Technologie eingesetzt werden, um die Freisetzung sowohl von AFS- oder Farbresten als auch von lebensfähigen adulten, juvenilen oder reproduktiven Stadien des Makrobewuchses zu minimieren. ${ }^{37}$ Das gesammelte Material soll in einer Weise entsorgt werden, die keine Gefahr für die aquatische Umwelt darstellt. $^{38}$

Für unter Wasser liegende Bereiche des Schiffes, die mit bioziden AFS beschichtet sind, sollen gem. Ziff. 7.8 S. 1 der Biofouling-Richtlinien Reinigungstechniken verwendet werden, die die Freisetzung von Biozid in die Umwelt mi- nimieren. Die Reinigung stark verschmutzter AFS könne nicht nur Biomasse erzeugen, sondern auch das AFS vorzeitig abtragen und Biozide freisetzen. ${ }^{39}$ Diese könnten wiederum die lokale Umwelt schädigen und sich auf künftige Vorhaben der Hafenbehörde zur Entsorgung von Baggergut aus dem Hafenbereich auswirken. ${ }^{40}$ Zudem würden abgenutzte AFS auf Schiffsrümpfen schnell wieder von Biofouling besiedelt. ${ }^{41}$ Die Unterwasserreinigung werde daher nicht zum Zweck eines Hinausschiebens der Wartung des Schiffes im Trockendock über die Lebensdauer des AFS hinaus empfohlen. ${ }^{42}$

In Ziff. 7.9 S. 1 der Biofouling-Richtlinien wird darauf hingewiesen, dass unter Wasser liegende Bereiche des Schiffes, die mit biozidfreien AFS beschichtet sind, möglicherweise eine regelmäßige Unterwasserreinigung als Teil der geplanten Wartung erfordern, um die Effizienz des Schiffsrumpfes aufrechtzuerhalten und das Risiko der Einführung invasiver aquatischer Arten zu minimieren. Auch hier sollen Reinigungstechniken verwendet werden, die das AFS nicht beschädigen und seine Funktion nicht beeinträchtigen. ${ }^{43}$

Was die Entsorgung der bei der Unterwasserreinigung anfallenden Stoffe anbelangt, so sollen gem. Ziff. 6.9 der Biofouling-Richtlinien Schiffswartungs- und Recyclinganlagen in Übereinstimmung mit den geltenden nationalen und lokalen Gesetzen und Vorschriften Maßnahmen ergreifen, um sicherzustellen, dass lebensfähige BiofoulingOrganismen oder chemische und physikalische Schadstoffe nicht in die aquatische Umwelt freigesetzt werden.

Sowohl Flaggenstaaten als auch Küstenstaaten können selbst bestimmen, ob und inwieweit sie die nicht verbindlichen Empfehlungen in den Biofouling-Richtlinien in ihrer Jurisdiktion für verbindlich erklären. ${ }^{44}$ Daher können aus ihnen unmittelbar keine Vorgaben für die nationale Regulierung der Unterwasserreinigung hergeleitet werden. Gleichzeitig können die Biofouling-Richtlinien aber beispielsweise die nationalen (Wasser-) Behörden bei ihren (Genehmigungs-) Entscheidungen leiten und wichtige

20) IMO, Guidelines for the Control and Management of Ships' Biofouling to Minimize the Transfer of Invasive Aquatic Species, 15. 7.2011, Res. MEPC.207(62). Siehe auch IMO, Guidance for Minimizing the Transfer of Invasive Aquatic Species as Biofouling (Hull Fouling) for Recreational Craft (12.11.2012), MEPC.1/Circ.792.

21) Für eine allgemeine Übersicht über die Biofouling-Richtlinien, siehe Schatz, Die Regulierung der Einführung invasiver gebietsfremder Arten durch Biofouling an der Schnittstelle zwischen Naturschutzrecht und Seeschifffahrtsrecht, NuR 2021, $361 \mathrm{ff}$.

22) Ziff. 7.1 S. 1 Biofouling-Richtlinien.

23) Ziff. 7.1 S. 2 Biofouling-Richtlinien.

24) Ziff. 7.5 S. 1 Biofouling-Richtlinien.

25) Ziff. 7.5 S. 3 Biofouling-Richtlinien.

26) Ziff. 7.5 S. 4 Biofouling-Richtlinien. Siehe dazu auch IMO, Guidelines for the Development of a Ship Energy Efficiency Management Plan (SEEMP), 28. 10.2018, Res. MEPC.282(70).

27) Ziff. 7.5 S. 5 Biofouling-Richtlinien.

28) Ziff. 7.5 S. 6 Biofouling-Richtlinien.

29) Ziff. 7.5 S. 2 Biofouling-Richtlinien.

30) Ziff. 7.6 S. 1 Biofouling-Richtlinien.

31) Ziff. 7.6 S. 2 Biofouling-Richtlinien.

32) Ziff. 7.6.1 Biofouling-Richtlinien.

33) Ziff. 7.6.2 Biofouling-Richtlinien.

34) Ziff. 7.6.3 Biofouling-Richtlinien.

35) Ziff. 7.6.4 Biofouling-Richtlinien.

36) Ziff. 7.7 S. 2 Biofouling-Richtlinien.

37) Ziff. 7.7 S. 3 Biofouling-Richtlinien.

38) Ziff. 7.7 S. 4 Biofouling-Richtlinien.

39) Ziff. 7.8 S. 2 Biofouling-Richtlinien.

40) Ziff. 7.8 S. 2 Biofouling-Richtlinien.

41) Ziff. 7.8 S. 3 Biofouling-Richtlinien.

42) Ziff. 7.8 S. 4 Biofouling-Richtlinien.

43) Ziff. 7.9 S. 2 Biofouling-Richtlinien.

44) Ziff. 3.1 S. 2 Biofouling-Richtlinien. 
Anhaltspunkte für sinnvolle Anforderungen und Praktiken bieten.

\section{3 Überarbeitung der IMO Biofouling-Richtlinien}

Die Biofouling-Richtlinien der IMO befinden sich seit mehreren Jahren in einem Überarbeitungsprozess. ${ }^{45}$ Das Thema wurde zuletzt bei der 8 . Session des Sub-Committee on Pollution Prevention and Response (PPR) der IMO im März 2021 behandelt. ${ }^{46}$ Diskutiert wurde insbesondere der jüngste Bericht der Korrespondenzgruppe zur Überarbeitung der Biofouling Richtlinien, der sich insbesondere mit der Struktur der überarbeiteten Biofouling-Richtlinien befasst und der trotz einzelner Meinungsverschiedenheiten im Hinblick auf Details die Unterwasserreinigung von Seeschiffen auch für die zukünftigen Richtlinien vorsieht. ${ }^{47}$ In einem weiteren Dokument hat Deutschland gemeinsam mit Finnland und Polen über die Ergebnisse des COMPLETE Projekts berichtet. ${ }^{48}$ Insbesondere wird der Entwurf einer Regionalen Biofouling Management Roadmap vorgelegt, die im Rahmen des COMPLETE Projekts entwickelt und der Baltic Marine Environment Protection Commission (Helsinki Commission, HELCOM) vorgelegt wurde. ${ }^{49}$ Der Entwurf geht relativ detailliert auf die Unterwasserreinigung und die damit verbundenen Gefahren für die Umwelt ein und mahnt Vorsichtsmaßnahmen sowie den Einsatz geeigneter Technologien an..$^{50}$

Ein wichtiger Streitpunkt, der im Rahmen der jüngsten PPR Sitzung thematisiert wurde, ist derzeit die Frage, ob die Unterwasserreinigung auch bei Macrofouling zum Einsatz kommen soll (statt einer Reinigung im Trockendock) und in welchem Umfang die bei der Reinigung anfallende Biomasse bei Microfouling und Macrofouling aufgefangen und landseitig entsorgt werden soll. ${ }^{51} \mathrm{Im}$ Ergebnis wurde die Korrespondenzgruppe zur Überarbeitung der Biofouling Richtlinien wiedereingesetzt und mit der Überarbeitung und Entwicklung der (neuen) Biofouling-Richtlinien beauftragt, wobei der bisherige Entwurf als Vorlage dienen soll. Zudem wurde das Marine Environment Protection Committee (MEPC) der IMO darum gebeten, den Zeitrahmen für die Fertigstellung der Überarbeitung bis 2023 zu verlängern.

\section{Vorgaben im nationalen Umweltrecht}

Im deutschen Recht existieren bisher keine speziellen gesetzlichen Bestimmungen zum Umgang mit Biofouling Management. ${ }^{52}$ Auch wenn die Unterwasserreinigung thematisch den Seeverkehr betrifft, unterfällt sie - wie im Folgenden aufgezeigt - nicht dem umweltbezogenen Binnen- und Seeschifffahrtsrecht. Stattdessen richtet sich die Zulässigkeit der Unterwasserreinigung in den oberirdischen Gewässern und Küstengewässern nach den allgemeinen Vorschriften des Wasserhaushaltsgesetzes (WHG) - und in der ausschließlichen Wirtschaftszone (AWZ) nach dem Hohe-See-Einbringungsgesetz (HSEG). Speziell für die Frage der Verwertung und Entsorgung spielt auch das Kreislaufwirtschaftsgesetz (KrWG) eine Rolle.

\subsection{Keine Anwendbarkeit des umweltbezogenen Schifffahrtsrechts}

Sowohl das Seeschifffahrtsrecht als auch das Binnenschifffahrtsrecht enthalten umweltbezogene Vorschriften, die nicht zuletzt wegen der teilweise existierenden Vorrangbestimmungen - vor den allgemeinen Vorschriften des deutschen Umweltrecht zu prüfen sind.

\subsubsection{Seeschifffahrtsrecht: SeeUmwVerhV}

Auf den Seeverkehr bezogene Umweltvorschriften finden sich in der See-Umweltverhaltensverordnung (SeeUmw-
VerhV). Die SeeUmwVerhV ist gem. ihres $₫ 3$ Abs. 1 Nr. 1 auf die Binnenwasserstraßen des Bundes anwendbar, soweit diese Seeschifffahrtsstraßen ${ }^{53}$ sind. ${ }^{54}$ Daneben ist sie gem. \$3 Abs. 1 Nr. 2 in den Seewasserstraßen des Bundes und gem. $\$ 3$ Abs. 1 Nr. 3 SeeUmwVerhV in der deutschen AWZ anwendbar. Im Kontext des Biofouling Managements ist darauf hinzuweisen, dass die SeeUmwVerhV auch das AFSÜbereinkommen umsetzt (vgl. $\$ \$ 1$ Abs. 2 lit. b, 16-17 SeeUmwVerhV). Die SeeUmwVerhV enthält jedoch keine Regelungen im Hinblick auf die Unterwasserreinigung.

Ohnehin fallen bei der Unterwasserreinigung nicht Stoffe an, die unmittelbar auf den Schiffsbetrieb als solchen zurückzuführen sind, sondern Abfälle, die der Wartung von Schiffen außerhalb des Normalbetriebs zuzurechnen sind. ${ }^{55}$ Vor diesem Hintergrund darf auch bezweifelt werden, dass die SeeUmwVerhV der richtige Standort für Spezialregelungen zur Unterwasserreinigung wäre. Es kann dennoch nicht ausgeschlossen werden, dass ggf. in Zukunft von der IMO verabschiedete Vorgaben in der SeeUmwVerhV umgesetzt werden, die - so wie die IMO Biofouling-Richtlinien - sozusagen als Annex zu Regelungen, die das Umweltverhalten

45) Siehe dazu IMO, Guidance for Evaluating the 2011 Guidelines for the Control and Management of Ships' Biofouling to Minimize the Transfer of Invasive Aquatic Species (13.6.2013), MEPC.1/Circ.811.

46) Vgl. Lloyd's Register, IMO Pollution Prevention and Response Eighth Session (PPR 8), Summary Report, 2021, S. 5 f.

47) IMO, Review of the 2011 Guidelines for the Control and Management of Ships' Biofouling to Minimize the Transfer of Invasive Aquatic Species (Resolution MEPC.207(62)), Report of the Correspondence Group on Review of the Biofouling Guidelines, Submitted by Norway, 14.12.2020.

48) IMO, Review of the 2011 Guidelines for the Control and Management of Ships' Biofouling to Minimize the Transfer of Invasive Aquatic Species (Resolution MEPC.207.(62)), Comments on the Report of the Correspondence Group on Review of the Biofouling Guidelines (PPR 8/4), Submitted by Finland, Germany and Poland, PPR 8/4/1, 20.1.2021.

49) HELCOM/OSPAR JTG Ballast: Proposal for a Regional Baltic Biofouling Management Roadmap v. 27.11.2020, Stand 14.3.2021, abrufbar unter: https://portal.helcom.fi/meetings/ TG\%20BALLAST\%2011-2020-763/MeetingDocuments/ 0702_Rev.\%201\%20Proposal\%20for\%20a\%20Regional\%20Baltic\%20Biofouling\%20Management\%20Roadmap.pdf. Siehe zu den relevanten HELCOM Prozessen bereits Schatz, Die Regulierung der Einführung invasiver gebietsfremder Arten durch Biofouling an der Schnittstelle zwischen Naturschutzrecht und Seeschifffahrtsrecht, NuR 2021, $361 \mathrm{ff}$.

50) HELCOM/OSPAR JTG Ballast: Proposal for a Regional Baltic Biofouling Management Roadmap v. 27.11.2020, Stand 14.3.2021, abrufbar unter: https://portal.helcom.fi/meetings/ TG\%20BALLAST\%2011-2020-763/MeetingDocuments/ 0702 Rev.\%201\%20Proposal\%20for\%20a\%20Regional\%20Baltic\%20Biofouling\%20Management $\% 20$ Roadmap.pdf, S. $8 \mathrm{ff}$; vgl. auch Comments by Finland, Germany and Poland: IMO, Review of the 2011 Guidelines for the Control and Management of Ships' Biofouling to Minimize the Transfer of Invasive Aquatic Species (Resolution MEPC.207.(62)), Comments on the Report of the Correspondence Group on Review of the Biofouling Guidelines (PPR 8/4), Submitted by Finland, Germany and Poland, PPR 8/4/1, 20.1.2021, Annex, S. $1 \mathrm{f}$.

51) Vgl. Lloyd's Register, IMO Pollution Prevention and Response Eighth Session (PPR 8), Summary Report, 2021, S. 5 f.

52) Schatz, Die Regulierung der Einführung invasiver gebietsfremder Arten durch Biofouling an der Schnittstelle zwischen Naturschutzrecht und Seeschifffahrtsrecht, NuR 2021, $361 \mathrm{ff}$.

53) Vgl. $\$ 1$ Abs. 1 der Seeschifffahrtsstraßen-Ordnung (SeeSchStrO)

54) Für eine Darstellung der wegerechtlichen Einordnung deutscher See- und Binnengewässer, siehe Proelß/Schatz, Rechtliche Vorgaben zum Umgang mit Schiffsabwasser: Völker-, unions- und nationalrechtliche Anforderungen an Einleitungen von ScrubberAbwasser, Ballastwasser und häuslichem Abwasser durch Schiffe, 2019, S. $65 \mathrm{ff}$.

55) Siehe dazu auch unten 4.1.2. 
von Schiffen betreffen, auch Vorgaben für die Unterwasserreinigung machen. ${ }^{56}$ Dabei ist aber zu bedenken, dass diese Regelungen gerade nicht in den meisten Häfen gelten würden, da es sich bei diesen regelmäßig um LandeswasserstraBen handelt, in denen die SeeUmwVerhV nicht gilt.

\subsubsection{Binnenschifffahrtsrecht:}

\section{Straßburger Abfallübereinkommen}

Im Bereich des umweltbezogenen Binnenschifffahrtsrechts kommt im Hinblick auf die Entsorgung der angefallenen Biomasse (und ggf. Farb- und biozide Beschichtungsreste) eine Anwendbarkeit des Straßburger Übereinkommens über die Sammlung, Abgabe und Annahme von Abfällen in der Rhein- und Binnenschifffahrt von 1996 (CDNI) ${ }^{57}$ bzw. des CDNI-Vertragsgesetzes und des CDNI-Ausführungsgesetzes in Betracht. ${ }^{58}$ Zweck des CDNI ist der Schutz der aquatischen Umwelt und die Verbesserung der Sicherheit in der Binnenschifffahrt durch die Vermeidung der Einleitung und Einbringung von schiffsbetriebsbedingten Abfällen. Räumlich ist das CDNI gem. seines Art. 2 auf alle in Anlage 1 zum CDNI genannten Wasserstraßen anwendbar, wozu in Deutschland ,[a]lle dem allgemeinen Verkehr dienenden Binnenwasserstraßen, mit Ausnahme des deutschen Teils des Bodensees und der Rheinstrecke oberhalb Rheinfelden“" gehören. ${ }^{59}$ Der sachliche Anwendungsbereich des CDNI erfasst alle Fahrzeuge i.S.d. Art. 1 lit. g) CDNI, wozu sowohl Binnenschiffe als auch Seeschiffe (vgl. Art. 1 lit. i CDNI) zählen, soweit sie auf Binnenwasserstraßen verkehren. ${ }^{60}$

Gemäß Art. 3 Abs. 1 CDNI gilt auf Wasserstraßen für Fahrzeuge ein Verbot, Schiffsabfälle einzubringen. Die Vertragsstaaten des CDNI sind gem. Art. 3 Abs. 2 CDNI zur Sicherstellung der Einhaltung dieses Verbotes verpflichtet. Gem. Art. 3 Abs. 3 CDNI sind Ausnahmen hiervon nur in Übereinstimmung mit Anlage 2 des CDNI und den dazugehörigen Anhängen zulässig. „Schiffsabfall“" schließt gem. Art. 1 lit. a) CDNI „Stoffe oder Gegenstände“ ein, ,,deren sich ihr Besitzer entledigt, entledigen will oder entledigen muss" und die in den litt. b) - f) von Art. 1 CDNI näher bestimmt sind.

Im Hinblick auf die bei der Unterwasserreinigung freigesetzte Biomasse könnte zunächst an ,Schiffsbetriebsabfall" i. S.d. Art. 1 lit. b) CDNI gedacht werden. Allerdings müsste es sich bei der Biomasse dann um Abfall handeln, der ,,bei Betrieb und Unterhaltung des Fahrzeugs an Bord““ entstanden ist. Das ist schon deswegen nicht der Fall, weil die Reinigung nicht an Bord stattfindet. Aus der Legaldefinition wird aber auch sonst deutlich, dass nur Abfälle erfasst sind, die auch wirklich beim Schiffsbetrieb und nicht etwa bei der Wartung von Schiffen außerhalb des Normalbetriebs entstehen. Vor diesem Hintergrund muss bezweifelt werden, dass es sich bei der Biomasse um ,,sonstige Schiffsbetriebsabfälle“ i.S.v. Art. 1 lit. e) CDNI handelt, deren Einbringung nach Art. 9.01 Abs. 1 Anlage 2 Teil C Kapitel IX des CDNI verboten ist. Schon die ausdrücklich genannten Kategorien ,,häusliches Abwasser, Hausmüll, Klärschlamm, Slops und übrige[r] Sonderabfall" weisen nach den Legaldefinitionen in Art. 8.01 Anlage 2 Teil C Kapitel VIII des CDNI einen klaren Bezug zum Schiffsbetrieb auf. Im Fall des ,übrigen Sonderabfalls“" nach Art. 8.01 lit. e) Anlage 2 Teil C Kapitel VIII des CDNI, bei dem es sich um einen Auffangtatbestand handelt, ${ }^{61}$ verweist die Legaldefinition (etwas zirkulär) auf ,,Schiffsbetriebsabfall“, der nicht von den anderen Kategorien von Schiffsbetriebsabfall erfasst ist.

Somit ist die bei der Unterwasserreinigung anfallende Biomasse nicht vom CDNI erfasst und daher auch nicht nach Art. 3 Abs. 1 CDNI i. V.m. Art. 9.01 Abs. 1 Anlage 2 Teil C Kapitel IX des CDNI verboten. ${ }^{62}$

\subsection{Oberirdische Gewässer und Küstengewässer: Wasserhaushaltsrecht}

In oberirdischen Gewässern und Küstengewässern richtet sich die Zulässigkeit der Unterwasserreinigung maßgeblich nach dem Wasserhaushaltsrecht. Zweck des WHG ist nach $\$ 1$ WHG der Schutz der Funktionen von Gewässern durch eine nachhaltige Bewirtschaftung derselben. ${ }^{63}$ Dabei dient das WHG im Hinblick auf den Schutz des ökologischen Gewässerzustands insbesondere auch der Umsetzung der Richtlinie (EG) 2000/60 zur Schaffung eines Ordnungsrahmens für Maßnahmen der Gemeinschaft im Bereich der Wasserpolitik (Wasserrahmenrichtlinie) ${ }^{64}$ in Deutschland. ${ }^{65}$ Im Hinblick auf die Meeresgewässer dient das WHG auch der Umsetzung der Richtlinie (EG) 2008/56 zur Schaffung eines Ordnungsrahmens für Maßnahmen der Gemeinschaft im Bereich der Meeresumwelt (MSRL). ${ }^{66} \mathrm{Im}$ Kontext der Unterwasserreinigung stellen sich in Bezug auf das Regelungsinstrumentarium des WHG vor allem Fragen nach der Erlaubnispflichtigkeit und nach Verbotstatbeständen, die das Einbringen von Stoffen in Gewässer angeht.

\subsubsection{Anwendungsbereich des WHG}

Gem. $\$ 2$ Abs. 1 S. 1 WHG findet das WHG auf die oberirdischen Gewässer (Nr. 1) und die Küstengewässer (Nr. 2) Anwendung, weshalb dort unter anderem die allgemeinen Vorschriften zur Bewirtschaftung von Gewässern (\$S 6-24 WHG) gelten. ${ }^{67}$ „Oberirdische Gewässer“ i.S. d. \$2 Abs. 1 Nr. 1 WHG sind gem. $\$ 3$ Nr. 1 WHG ,das ständig oder zeitweilig in Betten fließende oder stehende oder aus Quellen wild abfließende Wasser". Wo Seeschiffe die Unterläufe von schiffbaren Flüssen befahren, um im Inland gelegene Seehäfen (etwa Hamburg oder Bremen) anzufahren, be-

56) Siehe zur Möglichkeit der Aufnahme von Regelungen zu Biofouling Management in die SeeUmwVerhV: Schatz, Die Regulierung der Einführung invasiver gebietsfremder Arten durch Biofouling an der Schnittstelle zwischen Naturschutzrecht und Seeschifffahrtsrecht, NuR 2021, $361 \mathrm{ff}$.

57) Vertragsparteien des CDNI sind Belgien, Deutschland, Frankreich, Luxemburg, die Niederlande und die Schweiz.

58) Vgl. zum System des CDNI auch Proelß/Schatz, Rechtliche Vorgaben zum Umgang mit Schiffsabwasser: Völker-, unions- und nationalrechtliche Anforderungen an Einleitungen von Scrubber-Abwasser, Ballastwasser und häuslichem Abwasser durch Schiffe, 2019, 46-49.

59) Beschluss CDNI 2012-I-1, in Kraft getreten am 1.7.2012, Stand 18.9.2021, abrufbar unter https://www.cdni-iwt.org/wp-content/uploads/2017/10/Akten_2011-2012_de.pdf, umgesetzt durch die Dritte Verordnung zu dem Übereinkommen über die Sammlung, Abgabe und Annahme von Abfällen in der Rheinund Binnenschifffahrt v. 9.2.2015, BGBl. II S. 210.

60) Siehe auch Reimann, Binnenschiffahrt, Umweltrecht und Gewässerschutz im nationalen und internationalen Kontext, 1999, 52.

61) Vgl. Proelß/Schatz, Rechtliche Vorgaben zum Umgang mit Schiffsabwasser: Völker-, unions- und nationalrechtliche Anforderungen an Einleitungen von Scrubber-Abwasser, Ballastwasser und häuslichem Abwasser durch Schiffe, 2019, S. 47.

62) Selbst wenn das CDNI anwendbar wäre, würde es nicht das WHG verdrängen. Siehe dazu Proelß/Schatz, Rechtliche Vorgaben zum Umgang mit Schiffsabwasser: Völker-, unions- und nationalrechtliche Anforderungen an Einleitungen von ScrubberAbwasser, Ballastwasser und häuslichem Abwasser durch Schiffe, 2019, S. 74, 78.

63) Faßbender, in: Landmann/Rohmer, UmweltR, 93. EL August 2020, \1 WHG Rdnr. 21.

64) Richtlinie 2000/60/EG des Europäischen Parlaments und des Rates v. 23.10.2000 zur Schaffung eines Ordnungsrahmens für Maßnahmen der Gemeinschaft im Bereich der Wasserpolitik, AB1. 2000 L 327, S. 1.

65) Siehe dazu z.B. Reese, Die Wasserrahmenrichtlinie in der Umsetzungskrise: Fortbestehende Umsetzungsdefizite und rechtlicher Handlungsbedarf zur ökologischen Gewässerentwicklung, NVwZ 2018, 1592.

66) Richtlinie 2008/56/EG des Europäischen Parlaments und des Rates v. 17.6.2008 zur Schaffung eines Ordnungsrahmens für Maßnahmen der Gemeinschaft im Bereich der Meeresumwelt, AB1. 2008 L 164, S. 19.

67) Vgl. Czychowski/Reinhardt, WHG, 12. Aufl. 2019, §2 WHG Rdnr. 10. 
finden sie sich folglich in oberirdischen Gewässern. Wegerechtlich liegen in den oberirdischen Gewässern die Binnenwasserstraßen des Bundes ${ }^{68}$ und der Länder. Die „Küstengewässer“ umfassen nach $\$ 3$ Nr. 2 WHG „das Meer zwischen der Küstenlinie bei mittlerem Hochwasser oder zwischen der seewärtigen Begrenzung der oberirdischen Gewässer und der seewärtigen Begrenzung des Küstenmeeres" ". ${ }^{69}$ Damit erstrecken sich die Küstengewässer wegerechtlich auf die Seewasserstraßen des Bundes ${ }^{70}$ und der Länder. Da somit die deutschen Seehäfen allesamt im Geltungsbereich des WHG liegen, wird sich die Frage der Zulässigkeit einer Unterwasserreinigung von Seeschiffen in Zukunft besonders häufig aus Sicht des Wasserhaushaltsrechts stellen.

\subsubsection{Erlaubnispflichtigkeit und Erlaubnisfähigkeit nach dem WHG}

Für die Fragen der Erlaubnispflichtigkeit und Erlaubnisfähigkeit der Unterwasserreinigung ist entscheidend, wie genau der Reinigungsvorgang gestaltet ist und was dabei mit der angefallenen Biomasse geschieht.

$\mathrm{Zu}$ beachten ist zunächst die als repressives Verbot mit Erlaubnisvorbehalt ausgestaltete wasserhaushaltsrechtliche Erlaubnispflicht für Benutzungen wie das Einleiten oder Einbringen von Stoffen gem. \$\$8 Abs. 1 i. V.m. \$9 Abs. 1 Nr. 4 WHG. Unter den Begriff, „Stoff“ ist bei einer weiten, dem Sinn und Zweck des WHG entsprechenden Auslegung ,jede Materie zu fassen, die vor dem Einbringen oder Einleiten in das Gewässer in diesem nicht enthalten war, insoweit also ein „Fremdkörper“ ist" ${ }^{71}$ Unabhängig von der Frage, ob bei der Unterwasserreinigung auch in signifikantem Umfang umweltschädliche Beschichtungen oder Farbreste freigesetzt werden, ist jedenfalls die Biomasse selbst als „Stoff“" i.S.d. \$9 Abs. 1 Nr. 4 WHG zu qualifizieren. Kommt es bei der Unterwasserreinigung zu Einleitungen oder einem Einbringen von Stoffen, besteht also - im Ausgangspunkt - eine Erlaubnispflicht. ${ }^{72}$

Daran ändert im speziellen Fall Schleswig-Holsteins auch $\$ 13$ Abs. 1 Nr. 2 lit. d) LWG S-H nichts, der das betriebsbedingte Einbringen und Einleiten von Stoffen durch Schiffe in Küstengewässern als erlaubnisfreie Benutzung einstuft, wenn die Schiffe in Fahrt sind und soweit das Einbringen oder Einleiten durch ,,internationale oder supranationale Vorschriften zugelassen" ist. ${ }^{73}$ Weder handelt es sich bei der Unterwasserreinigung um unmittelbaren Schiffsbetrieb, noch ist das Einbringen oder Einleiten der dabei anfallenden Stoffe durch völker- oder unionsrechtliche Regelungen oder deren Umsetzung im deutschen Recht zugelassen. ${ }^{74}$

Im Hinblick auf $₫ 9$ Abs. 1 Nr. 4 WHG ist zwischen einem Einbringen und einer Einleitung zu unterscheiden, denn das Einbringen „fester Stoffe“ zu Entledigungszwecken ist nach $\$ 32$ Abs. $1 \mathrm{~S}$. 1 WHG in oberirdischen Gewässern ${ }^{75}$ und nach $₫ 45$ Abs. 1 S. 1 WHG in Küstengewässern verboten. ${ }^{76}$ Im Vergleich zu flüssigen und gasförmigen Stoffen sind feste Stoffe in ihrer Form beständig und lassen sich nur schwer aufteilen, verformen oder komprimieren. ${ }^{77}$ Die beim Entfernen von Biofouling abgetragene Biomasse besteht regelmäßig - je nach Fortschritt des Bewuchses zumindest auch aus festen organischen Stoffen tierischen und/oder pflanzlichen Ursprungs. Es handelt sich also regelmäßig (auch) um ,feste Stoffe“".$^{78}$

Ein Stoff gilt nach $\$ \$ 32$ Abs. 1 S. 1, 45 Abs. 1 S. 1 WHG als ,eingebracht“, wenn er ,im Wege einer objektiv auf die Inanspruchnahme des Gewässers zweckgerichteten Zuführung fester Stoffe" in das Gewässer gelangt ist. ${ }^{79}$ Die Tatsache, dass ein fester Stoff bereits im Gewässer bzw. im Kontext von Biofouling jedenfalls im ständigen Kontakt mit dem Gewässer war, bevor er endgültig dem Gewässer zugeführt wurde, steht einer Qualifizierung als ,Einbringen“ i. S.d. $\iint 32$ Abs. 1 S. 1, 45 Abs. 1 S. 1 WHG nicht entgegen. ${ }^{80}$ Somit ist diese Voraussetzung der Unterwasserreini- gung erfüllt, wenn die freigesetzten Stoffe nicht aufgefangen, sondern im Wasser belassen werden. Dass die Biomasse zu Entledigungszwecken i.S.d. S\$ 32 Abs. 1 S. 1, 45 Abs. 1 S. 1 WHG, also zur dauerhaften Aufgabe der Sachherrschaft, in das Wasser eingebracht wird, ist evident. ${ }^{81}$ Somit ist eine Unterwasserreinigung unter Einsatz von Methoden bzw. Technologien verboten, die die freigesetzten festen Stoffe nicht vollständig aufzufangen in der Lage ist.

Demgegenüber ist bei einer Unterwasserreinigung auch ein Einleiten i.S.d. $\$ 9$ Abs. 1 Nr. 4 Alt. 2 WHG technisch denkbar, wenn etwa zuvor abgepumptes und filtriertes Wasser (ggf. mit Resten der Biomasse - insbesondere des Microfoulings) in flüssiger Form (etwa über eine Leitung) dem Wasser zugeführt wird. ${ }^{82}$ Wird ein solches Vorgehen als Einleitung klassifiziert, so ist diese gem. $\$ 8$ Abs. 1 WHG erlaubnispflichtig, je nach konkreter Ausgestaltung aber zusätzlich dem strengen Regime für Einleitungen von Abwasser gem. \57 Abs. 1 WHG unterworfen.

Speziell in oberirdischen Gewässern kommt schließlich noch eine Erlaubnis- bzw. Bewilligungspflicht der Unterwasserreinigung gem. IS 8 Abs. 1,9 Abs. 1 Nr. 1 Alt. 1 WHG in Betracht, sofern durch die eingesetzte Technik Wasser abgepumpt und damit dem oberirdischen Gewässer dauerhaft entzogen wird. ${ }^{83}$

68) Vgl. $\$ 1$ Abs. 1 Nr. 1 WaStrG. Siehe dazu Reinhardt/Schäfer, in: Schäfer, Bundeswasserstraßengesetz, 3. Aufl. 2017, \$1 WaStrG Rdnr. $1 \mathrm{f}$.

69) Die seewärtige Begrenzung von oberirdischen Gewässern, die nicht Binnenwasserstraßen des Bundes sind, richtet sich dabei nach den landesrechtlichen Vorschriften.

70) Vgl. $\ 1$ Abs. 1 Nr. 2 WaStrG. Siehe dazu Reinhardt/Schäfer, in: Schäfer, Bundeswasserstraßengesetz, 3. Aufl. 2017, \$1 WaStrG Rdnr. 3.

71) Pape, in: Landmann/Rohmer, UmweltR, 93. EL August 2020 , \$9 WHG Rdnr. 44.

72) Eine Bewilligung scheidet insoweit gem. \14 Abs. 1 Nr. 3 WHG aus. Vgl. Pape, in: Landmann/Rohmer, UmweltR, 93. EL August 2020, \$9 WHG Rdnr. 43.

73) Vgl. zu dieser Norm Mohr, in: Kollmann/Mohr, Wassergesetz des Landes Schleswig-Holstein (Landeswassergesetz - LWG), Januar 2020, \$13 LWG Rdnr. 3.5; Proelß, in: Landmann/Rohmer, UmweltR, 93. EL August 2020, $\$ 43$ WHG Rdnr. 45.

74) Siehe oben 4.1

75) Siehe zum Anwendungsbereich der Vorschrift Faßbender, in: Landmann/Rohmer, UmweltR, 93. EL August 2020, $\$ 32$ WHG Rdnr. 4 ff.

76) Vgl. dazu Czychowski/Reinhardt, WHG, 12. Aufl. 2019, $₫ 45$ WHG Rdnr. 5 ff. Dieses Verbot dient der Umsetzung u.a. des Londoner Protokolls. Siehe dazu Proelß, in: Landmann/Rohmer, UmweltR, 93. EL August 2020, 445 WHG Rdnr. 10.

77) Posser, in Giesberts/Reinhardt, BeckOK UmweltR, 57. Ed. 1.7.2020, $\$ 32$ WHG Rdnr. 12f.; Czychowski/Reinhardt, WHG, 12. Aufl. 2019, \$32 WHG Rdnr. 7; Faßbender, in: Landmann/ Rohmer, UmweltR, 93. EL August 2020, §32 WHG Rdnr. 11.

78) Vgl. auch Czychowski/Reinhardt, WHG, 12. Aufl. 2019, \32 WHG Rdnr. 6: „Tierkadaver, tote Fische, Rechengut von Stauanlagen".

79) Proelß, in: Landmann/Rohmer, UmweltR, 93. EL August 2020 , $\$ 45$ WHG Rdnr. 11, m.w. N. aus Rechtsprechung und Literatur. Vgl. auch Czychowski/Reinhardt, WHG, 12. Aufl. 2019, \$32 Rdnr. 6 f.

80) Vgl. mit Verweis auf den Umkehrschluss aus $₫ 45$ Abs. 1 S. 2 WHG: Proel $\beta$, in: Landmann/Rohmer, UmweltR, 93. EL August 2020, \45 Rdnr. 11; Rossi, in: Sieder/Zeitler/Dahme/Knopp, WHG AbwAG, 53. EL August 2019, \45 WHG Rdnr. 33; Faßbender, in: Landmann/Rohmer, UmweltR, 93. EL August 2020, $\$ 32$ Rdnr. 13.

81) Vgl. zur Definition Czychowski/Reinhardt, WHG, 12. Aufl. 2019 , $\$ 32$ WHG Rdnr. 9.

82) Siehe zum Begriff des Einleitens etwa Pape, in: Landmann/ Rohmer, UmweltR, 93. EL August 2020, \$9 WHG Rdnr. 50 f.

83) Vgl. zu diesem Tatbestand allgemein Knopp, in: Sieder/Zeitler/ Dahme/Knopp, WHG AbwAG, 53. EL August 2019, \$9 WHG Rdnr. $23 \mathrm{f}$. 
Schlussendlich ist es zumindest hypothetisch denkbar, dass der Vorgang der Unterwasserreinigung technisch so gestaltet werden kann, dass dabei sämtliche Biomasse und Schadstoffe aufgefangen werden, ohne dass es zu einem Einbringen, einer Einleitung oder (in oberirdischen Gewässern) zu einer dauerhaften Entnahme von Wasser kommt. In diesem Fall wäre - für den dann isoliert zu betrachtenden Reinigungsvorgang im Wasser - kein nach $₫ 8$ Abs. 1 WHG erlaubnispflichtiger Benutzungstatbestand gem. \$9 Abs. 1 oder 2 WHG ersichtlich. Es ist freilich fraglich, ob eine solche Unterwasserreinigung technisch möglich ist. Zudem ist zu bedenken, dass den zuständigen Wasserbehörden in diesem Fall das präventive Kontrollinstrument des Erlaubnisverfahrens genommen würde und nur repressiv durch aufwändige Kontrollen sichergestellt werden könnte, dass die eingesetzten Methoden auch wirklich nicht zu problematischen Einträgen von Biomasse oder Schadstoffen führen. In der Praxis wäre dies wegen des hohen Vollzugsaufwands kaum umsetzbar.

\subsection{Deutsche AWZ: Hohe-See-Einbringungsgesetz}

In der deutschen AWZ ist für die Frage der rechtlichen Rahmenbedingungen der Unterwasserreinigung nicht das WHG, sondern das HSEG maßgeblich. Das HSEG dient gem. \$1 HSEG der „Erhaltung der Meeresumwelt sowie deren Schutz vor Verschmutzung durch das Einbringen von Abfällen oder anderen Stoffen und Gegenständen." Insoweit bezweckt es vor allem die Umsetzung des Londoner Übereinkommens und des Londoner Protokolls im deutschen Recht. ${ }^{84}$ Derzeit ist noch nicht absehbar, dass für Seeschiffe in großem Umfang Unterwasserreinigungen in der AWZ durchgeführt werden. Dennoch sind solche Aktivitäten sowohl für auf die Hafeneinfahrt wartende Schiffe als auch für Plattformen und sonstige auf See errichtete Anlagen denkbar.

\subsubsection{Anwendungsbereich des HSEG} in Abgrenzung zum WHG

Das HSEG gilt nach $\$ 2$ Abs. 1 S. 1 HSEG in der „Hohen See“, wobei die Legaldefinition dieses Begriffes nicht nur die Hohe See im völkerrechtlichen Sinne umfasst, sondern ,alle Meeresgewässer mit Ausnahme des Küstenmeeres unter deutscher Souveränität sowie der Küstenmeere unter der Souveränität anderer Staaten“. Nach $\$ 2$ Abs. 1 S. 2 HSEG gehören hierzu ausdrücklich auch die AWZ und der Meeresboden. Sachlich gilt das HSEG unter anderem gem. \2 Abs. 2 Nr. 1 HSEG für ,Schiffe, Luftfahrzeuge, Plattformen oder sonstige auf See errichtete Anlagen", 85 die sich in der deutschen AWZ befinden. Eine Ausnahme gilt nach \2 Abs. 3 HSEG für Schiffe und Luftfahrzeuge der Bundeswehr.

Auch der Begriff der „Meeresgewässer“ gem. \$3 Nr. 2a WHG schließt neben den Küstengewässern ,,die Gewässer im Bereich der deutschen ausschließlichen Wirtschaftszone und des Festlandsockels, jeweils einschließlich des Meeresgrundes und des Meeresuntergrundes“ ein. Das WHG enthält allerdings keine Regelungen, die für eine Unterwasserreinigung in der deutschen AWZ maßgeblich sind, da für Meeresgewässer gem. $\$ 2$ Abs. 1a WHG mit Ausnahme der Küstengewässer nur die Vorschriften des $\$ 23$, des Kapitels 2 Abschnitt $3 \mathrm{a}$ und des $\$ 90$ WHG anwendbar sind. ${ }^{86}$

4.3.2 Verbot des Einbringens von Abfällen oder sonstigen Stoffen nach dem HSEG

Nach $₫ 4$ S. 1 HSEG ist das Einbringen von Abfällen und sonstigen Stoffen und Gegenständen in die Hohe See verboten. Gleiches gilt gem. \6 HSEG für die Verbrennung von Abfällen und sonstigen Stoffen auf Hoher See. Nach \4 S. 2 HSEG gelten vom Verbot der Einbringung bestimmte abschließend aufgezählte (vorliegend nicht einschlägige) Ausnahmen, die jeweils gem. $\$ 5$ HSEG erlaubnispflichtig sind.
Die Legaldefinition des Begriffs „Einbringen“ in $\$ 3$ Abs. 1 S. 1 HSEG nennt fünf verschiedene Varianten, wobei vorliegend ,,jede in die Hohe See erfolgende Beseitigung von Abfällen oder sonstigen Stoffen von Schiffen, Luftfahrzeugen, Plattformen oder sonstigen auf See errichteten Anlagen aus" ( $\$ 3$ Abs. 1 S. 1 Nr. 1 HSEG) einschlägig ist. Demgegenüber ist „Verbrennung auf See“"gem. \$3 Abs. 2 HSEG „,die Verbrennung von Abfällen oder sonstigen Stoffen zum Zwecke ihrer vorsätzlichen Beseitigung durch Wärmezerstörung an Bord eines Schiffes, einer Plattform oder eines sonstigen auf Hoher See errichteten Bauwerks."

Das HSEG enthält keine Legaldefinition der Begriffe „Abfälle“ oder „,sonstige Stoffe“. Allerdings kann insoweit hilfsweise auf Art. 1.8 des Londoner Protokolls zurückgegriffen werden, nach dem „Gegenstände und Stoffe jeder Art, jeder Form und jedes Typs“" unter den Begriff „Abfälle oder sonstige Stoffe" fallen. ${ }^{87}$ Ausschlaggebend ist - wie im Abfallrecht üblich - die Entledigungsabsicht. Biomasse und Beschichtungs- bzw. Farbreste, wie sie bei der Unterwasserreinigung anfallen, sind vor diesem Hintergrund als „Abfälle oder sonstige Stoffe und Gegenstände“ zu qualifizieren. Somit ist die Unterwasserreinigung auch in der AWZ nur zulässig, wenn eine Methode bzw. Technologie eingesetzt wird, bei der die Biomasse samt Farb- und Beschichtungsresten vollständig aufgefangen und nicht im Wasser entsorgt wird.

Es steht hier auch kein Einbringen schiffsbetriebsbezogener Abfälle oder Stoffe in Rede, ${ }^{88}$ weshalb die Frage des Vorranges u.a. des MARPOL-Übereinkommens und seiner Anlagen gegenüber dem HSEG gem. \12 Nr. 3 HSEG hier nicht diskutiert werden muss. ${ }^{89}$ Auch der Vorrang des umweltbezogenen Seeschifffahrtsrechts, der sich aus dem Verweis auf das Seeaufgabengesetz (SeeAufgG) in $\$ 12$ Nr. 4 HSEG ergibt, kommt hier nicht zum Zug. Solche auf den Seeverkehr bezogene Umweltvorschriften, die in der deutschen AWZ gelten, finden sich in der SeeUmwVerhV, die aber - wie bereits festgestellt - vorliegend nicht weiterhilft. ${ }^{90}$

\subsection{Verwertung oder Entsorgung der Biomasse nach dem $\mathrm{KrWG}$}

Das KrWG spielt für die Frage der Zulässigkeit eines Einbringens der bei der Unterwasserreinigung anfallenden Biomasse keine eigenständige Rolle, wohl aber im Hinblick auf deren Entsorgung an Land.

4.4.1 Vorrang des WHG gegenüber dem KrWG in oberirdischen Gewässern und Küstengewässern

Beim Einbringen von Stoffen in Gewässer muss stets an den in $\$ 2$ Abs. $2 \mathrm{Nr}$. $9 \mathrm{KrWG}$ niedergelegten Vorrang des in Bezug auf das Umweltmedium Wasser spezielleren Gewäs-

84) Vgl. BT-Drs. 13/10364, S. 1.

85) Vgl. zur Legaldefinition von „Schiffen und Luftfahrzeugen“ $\$ 3$ Abs. 3 HSEG: „Wasserfahrzeuge und Fluggeräte jeder Art. Hierzu gehören auch Luftkissenfahrzeuge und schwimmendes Gerät mit oder ohne Eigenantrieb."

86) Vgl. etwa Faßbender, in: Landmann/Rohmer, UmweltR, 93. EL August 2020, \2 WHG Rdnr. 37a; Czychowski/Reinhardt, WHG, 12. Aufl. 2019, \2 Rdnr. 12b; Weiß, in: Giesberts/Reinhardt, BeckOK UmweltR, 57. Ed. 1.7.2020, §45a WHG Rdnr. 2.

87) Zur Möglichkeit des Rückgriffs auf die einschlägigen völkerrechtlichen Abkommen bei der Auslegung offener Rechtsbegriffe im HSEG: Spoerr, in: Versteyl/Mann/Schomerus, Kreislaufwirtschaftsgesetz: KrWG, 4. Aufl. 2014, \$29 KrWG Rdnr. 70.

88) Vgl. oben 4.1.1, 4.1.2.

89) Siehe dazu Spoerr, in: Versteyl/Mann/Schomerus, Kreislaufwirtschaftsgesetz: KrWG, 4. Aufl. 2014, \$29 KrWG Rdnr. 72; Proelß/Schatz, Rechtliche Vorgaben zum Umgang mit Schiffsabwasser: Völker-, unions- und nationalrechtliche Anforderungen an Einleitungen von Scrubber-Abwasser, Ballastwasser und häuslichem Abwasser durch Schiffe, 2019, S. $78 \mathrm{ff}$.

90) Siehe oben 4.1.1. 
serschutzrechts vor dem Kreislaufwirtschaftsrecht gedacht werden. ${ }^{91}$ Diese Norm dient der Abgrenzung der beiden Rechtsregime, ${ }^{92}$ die sich sonst wegen der jeweils weit gefassten Abfall- und Abwasserbegriffe erheblich überschneiden würden. ${ }^{93}$

Bei der Anwendung von $\$ 2$ Abs. 2 Nr. 9 KrWG ist der Gewässerbegriff des WHG zugrunde zu legen, da die Anwendbarkeit der allgemeinen Regelungen des WHG an diesen anknüpft. ${ }^{94}$ Für das Einbringen von Stoffen in oberirdische Gewässer und Küstengewässer ist also gem. \2 Abs. 2 Nr. 9 KrWG das WHG vorrangig. ${ }^{95}$ Da $\int S 32$ Abs. 1 S. 1, 45 Abs. 1 S. 1 WHG das Einbringen von festen Stoffen in oberirdische Gewässer und Küstengewässer verbieten und die angefallene Biomasse somit nicht unmittelbar im Wasser entsorgt werden darf, bleibt für das KrWG nur eine Rolle bei der späteren Verwertung oder Entsorgung der Biomasse an Land. ${ }^{96}$

\subsubsection{Vorrang des HSEG gegenüber} dem KrWG in der AWZ

In der AWZ stellt sich die rechtliche Situation im Ausgangspunkt, nicht aber im Ergebnis, anders dar. Da $\$ 2$ Abs. 1 S. 1 WHG den Begriff der „Gewässer“, für die das WHG insgesamt gilt, nicht auf die Meeresgewässer jenseits der Küstengewässer ausdehnt, ${ }^{97}$ finden die allgemeinen wasserhaushaltsrechtlichen Regelungen keine Anwendung. ${ }^{98}$ Damit ist $\$ 2$ Abs. 2 Nr. 9 KrWG nicht auf das Einbringen von Abfällen in die AWZ anwendbar. ${ }^{99} \mathrm{Da}$ es sich bei der Biomasse, die bei der Unterwasserreinigung anfällt, nicht um Schiffsabfälle handelt, ${ }^{100}$ greift auch nicht $\$ 2$ Abs. 2 Nr. $13 \mathrm{KrWG}$, der unter anderem das Konkurrenzverhältnis des KrWG zum MARPOL-Übereinkommen regelt. ${ }^{101}$

Die grundsätzliche Anwendbarkeit des KrWG auf das Einbringen von Abfällen und sonstigen Stoffen in der AWZ wird wiederum auch aus $\$ 29$ Abs. 4 S. 1 KrWG deutlich, der unter Verweis auf das HSEG das Einbringen von Abfällen in die AWZ sowie die Verbrennung von Abfällen in der AWZ verbietet. Ebenso impliziert der Verweis auf das HSEG, dass die vom HSEG erfassten Abfälle und sonstigen Stoffe auch vom - ohnehin sehr weit gefassten - Abfallbegriff des $₫ 3$ Abs. 1 S. 1 KrWG erfasst sind..$^{102}$ Jedoch gilt das Verbot „nach Maßgabe“ des HSEG, womit es letztlich auf den räumlichen und sachlichen Geltungsbereich des HSEG und ggf. im HSEG bestehende Ausnahmen ankommt. ${ }^{103}$ Während also das HSEG die Entsorgung der durch eine Unterwasserreinigung freigesetzten Biomasse in der AWZ verbietet, ist die Rolle des KrWG letztlich auf die Frage der Verwertung oder Entsorgung der aufzufangenden Biomasse beschränkt.

\section{Schlussbetrachtung}

Die Unterwasserreinigung von Seeschiffen dient der Vorbeugung der Einführung gebietsfremder invasiver Arten durch Biofouling (sowie indirekt der Reduzierung von schiffsbetriebsbedingten Emissionen), kann aber ihrerseits zu Gewässerverschmutzung führen, wenn die dabei freigesetzte Biomasse samt bioziden Beschichtungs- und Farbresten nicht sachgerecht aufgefangen und entsorgt wird. Auch wenn auf internationaler Ebene bisher keine verbindlichen Regelungen speziell für die Unterwasserreinigung existieren, liefern die IMO Biofouling-Richtlinien und die im Kontext des Überarbeitungsprozesses dieser Richtlinien erarbeiteten Empfehlungen hilfreiche Anhaltspunkte für die deutsche Behördenpraxis. In Deutschland lässt sich die Problematik zumindest teilweise über das Wasserhaushaltsrecht und das HSEG auffangen. Das umweltbezogene (Binnen-) und Seeschifffahrtsrecht ist demgegenüber nicht auf die Unterwasserreinigung bzw. die Frage der Zulässigkeit eines Einbringens der dabei anfallenden Biomasse anwendbar.
Im Ergebnis hängt die wasserhaushaltsrechtliche Erlaubnispflichtigkeit und Erlaubnisfähigkeit der Unterwasserreinigung von Seeschiffen in oberirdischen Gewässern und Küstengewässern davon $a b$, was für eine Methode bzw. Technologie angewandt wird. Werden beim Reinigungsvorgang feste Stoffe in das Wasser eingebracht, so ist die Unterwasserreinigung ausnahmslos verboten und auch nicht erlaubnisfähig. Erfüllt die eingesetzte Methode einen Einleitungstatbestand, so ist die Unterwasserreinigung erlaubnispflichtig und nur unter Einhaltung der übrigen Voraussetzungen (etwa für die Einleitung von Schmutzwasser) auch erlaubnisfähig. In oberirdischen Gewässern (also insbesondere in den an Flüssen gelegenen Seehäfen) kann die Unterwasserreinigung auch dann erlaubnispflichtig sein, wenn dabei Wasser dauerhaft entnommen wird. Werden - hypothetisch - beim Reinigungsvorgang hingegen keinerlei feste Stoffe freigesetzt und auch kein Wasser (wieder) eingeleitet oder dauerhaft entnommen, so ist die Unterwasserreinigung nicht nach dem WHG erlaubnispflichtig. $\mathrm{Ob}$ eine solche Reinigung technisch möglich und vor dem Hintergrund der dann wegfallenden Erlaubnispflicht im Ergebnis wünschenswert ist, steht freilich auf einem anderen Blatt. In der deutschen AWZ muss bei einer Unterwasserreinigung derzeit (nur) das absolute Verbot des Einbringens der durch die Reinigung freigesetzten festen Stoffe und das Verbot des Verbrennens beachtet werden. Daher bietet sich in der AWZ im Grundsatz ein Einsatz der gleichen Reinigungstechnik wie in anderen Gewässern an.

Ist ein wasserhaushaltsrechtlicher Erlaubnistatbestand einschlägig, so haben die jeweils nach dem Landesrecht zuständigen Behörden die Möglichkeit, die konkreten zur Anwendung gelangenden Reinigungsmethoden und -technologien zu überprüfen und Anforderungen an deren Einsatz zu stellen. In diesem Rahmen dürften eine belastbare Risikobewertung sowie nach Bedarf Auflagen und Bedingungen für die Reinigung $\mathrm{zu}$ fordern sein. In diesem Rahmen sollten die Behörden Empfehlungen wie die IMO Biofouling-Richtlinien ${ }^{104}$ sowie die Einschätzung der $\mathrm{Na}$ turschutzbehörden zur Frage der möglicherweise in der Biomasse enthaltenen lebensfähigen Neobiota zu berück-

91) Gesetzesbegründung, BT-Drs. 17/6052, S. 60; Beckmann, Landmann/Rohmer, UmweltR, 93. EL August 2020, \$2 KrWG Rdnr. 86.

92) Instruktiv Scheier, Die Nichtanwendung des KrWG im Verhältnis zum Wasser- und Bodenrecht, UPR 2011, 300.

93) Siehe dazu und auch zu unionsrechtlichen Bedenken gegen $₫ 2$ Abs. 2 Nr. 9 KrWG die Diskussion bei Beckmann, in: Landmann/Rohmer, UmweltR, 93. EL August 2020, $\$ 2$ KrWG Rdnr. 86.

94) Vgl. Wolf, in: Giesberts/Reinhardt, BeckOK UmweltR, 57. Ed. 1.7.2020, $\$ 2$ KrWG Rdnr. 43.

95) Vgl. Beckmann, in: Landmann/Rohmer, UmweltR, 93. EL August 2020, $\$ 2$ KrWG Rdnr. 88.

96) Vgl. Petersen, in: Jarass/Petersen, Kreislaufwirtschaftsgesetz, 2014, \$2 KrWG Rdnr. 102.

97) Siehe oben 4.3.1.

98) Vgl. Guckelberger, in: Giesberts/Reinhardt, BeckOK UmweltR, 57. Ed. 1.7.2020, $\$ 2$ WHG Rdnr. 7. Unzutreffend daher Petersen, in: Jarass/Petersen, Kreislaufwirtschaftsgesetz, 2014, \2 KrWG Rdnr. 96.

99) Kropp, in: Giesberts/Reinhardt, BeckOK UmweltR, 57. Ed. 1.7.2020, \$29 KrWG Rdnr. 45; Spoerr, in: Jarass/Petersen, Kreislaufwirtschaftsgesetz, 2014, $\$ 29$ KrWG Rdnr. 77.

100) Vgl. oben 4.1.1.

101) Vgl. zum Anwendungsbereich von $₫ 2$ Abs. 2 Nr. 13 KrWG auch Petersen, in: Jarass/Petersen, Kreislaufwirtschaftsgesetz, 2014, \$2 KrWG Rdnr. $132 \mathrm{ff}$.

102) Vgl. zum Abfallbegriff allgemein Beckmann, in: Landmann/ Rohmer, UmweltR, 93. EL August 2020, \$3 KrWG Rdnr. $8 \mathrm{ff}$

103) Vgl. Kropp, in: Giesberts/Reinhardt, BeckOK UmweltR, 57. Ed. 1.7.2020, KrWG $\$ 29$ Rdnr. 46.

104) Siehe oben 3.2 . 
sichtigen. ${ }^{105}$ Zur Vermeidung von Rechtsunsicherheit für die Schifffahrt und zur Reduzierung der Gefahr einer Umgehung von Umweltstandards sollten bundesweit einheitliche Standards angestrebt werden. In jedem Fall ist ein Einbringen der Biomasse in das Wasser verboten, weshalb diese im Einklang mit dem KrWG an Land verwertet oder entsorgt werden muss.

Open Access. Dieser Artikel wird unter der Creative Commons Namensnennung 4.0 International Lizenz veröffentlicht, welche die Nutzung, Vervielfältigung, Bearbeitung, Verbreitung und Wiedergabe in jeglichem Medium und Format erlaubt, sofern Sie den/die ursprünglichen Autor(en) und die Quelle ordnungsgemäß nennen, einen Link zur Creative Commons Lizenz beifügen und angeben, ob Änderungen vorgenommen wurden.

Die in diesem Artikel enthaltenen Bilder und sonstiges Drittmaterial unterliegen ebenfalls der genannten Creative Commons Lizenz, so- fern sich aus der Abbildungslegende nichts anderes ergibt. Sofern das betreffende Material nicht unter der genannten Creative Commons Lizenz steht und die betreffende Handlung nicht nach gesetzlichen Vorschriften erlaubt ist, ist für die oben aufgeführten Weiterverwendungen des Materials die Einwilligung des jeweiligen Rechteinhabers einzuholen.

Weitere Details zur Lizenz entnehmen Sie bitte der Lizenzinformation auf http://creativecommons.org/licenses/by/4.0/deed.de.

Open Access funding enabled and organized by Projekt DEAL.

105) Vgl. zum insoweit einschlägigen Naturschutzrecht Schatz, Die Regulierung der Einführung invasiver gebietsfremder Arten durch Biofouling an der Schnittstelle zwischen Naturschutzrecht und Seeschifffahrtsrecht, NuR 2021, $361 \mathrm{ff}$.

\title{
Der Abschneidewert für Stickstoffeinträge im Habitatschutz
}

\author{
Friedrich Hacker, Florian Jansen, Thomas Krämerkämper, Peter Kremer, Dirk Tessmer
}

(C) Der/die Autor(en) 2021. Dieser Artikel ist eine Open-Access-Publikation.

Die Auswirkungen von Stickstoffeinträgen in FFH-Gebiete sind eine der zentralen fachlichen und rechtlichen Fragestellungen im Habitatschutz. Das BVerwG hat hierzu - auf der Grundlage fachlicher Leitfäden - seine Rechtsprechung zu Bagatell-/Irrelevanzschwellen und einem Abschneidekriterium entwickelt. Während Bagatell-/Irrelevanzschwellen an der Empfindlichkeit von Lebensräumen orientiert sind, ist das Abschneidekriterium (in Höhe von $0,3 \mathrm{~kg} \mathrm{~N} /($ ha $\star a)$ ) ein $\mathrm{ab}-$ soluter Wert; liegt der Eintrag aus einem Projekt darunter, muss nicht weiter geprüft werden. Auch summierende Einträge unterhalb dieser Schwelle werden nicht berücksichtigt.

Im Folgenden wird - nach einem Überblick über die Rechtsprechung - gezeigt, dass die naturwissenschaftlichen Annahmen, die der rechtlichen Einordnung des BVerwG zugrunde liegen, nicht haltbar sind. Teilweise sind die fachlichen Aussagen nicht hergeleitet oder nachgewiesen (z. B. die untere Wirkungsschwelle), teilweise sind sie falsch (z.B. die Untergrenze der Messbarkeit oder die fehlende Zuordenbarkeit zu einem Emittenten). Es wird weiter gezeigt, dass von Einträgen unterhalb von $0,3 \mathrm{~kg} \mathrm{~N} /\left(\mathrm{ha}^{\star} \mathrm{a}\right)$ schädliche Wirkungen ausgehen können, was dann im Einzelfall untersucht werden muss.

Ein absoluter Abschneidekriterium in Höhe von 0,3 kg N/ (ha $\star_{a}$ ) kann daher vor dem Hintergrund, dass im Habitatschutzrecht vernünftige Zweifel ausgeschlossen sein müssen, keinen Bestand haben. Die vom BVerwG herangezogenen Leitfäden können nicht (mehr) als Fachkonventionen angesehen werden.

\section{Dipl.-Biol. Friedrich Hacker, Diplom-Biologe,}

Greifswald, Deutschland

Prof. Dr. rer. nat Florian Jansen,

Prof. für Landschaftsökologie und Standortkunde, Uni Rostock, Rostock, Deutschland

Dr. rer. nat. Thomas Krämerkämper, Diplom-Physiker, Castrop-Rauxel, Deutschland

Peter Kremer, Rechtsanwalt und Fachanwalt für Verwaltungsrecht, Berlin, Deutschland

Dirk Tessmer, Rechtsanwalt,

Frankfurt a. M., Deutschland

\section{Stickstoffeinträge als naturschutzfachliche und rechtliche Herausforderung}

Mit seinem Globalen Bericht zum Zustand der Natur hat der Weltbiodiversitätsrat (IPBES) eindringlich darauf aufmerksam gemacht, dass weltweit bis zu einer Million Tier- und Pflanzenarten vom Aussterben bedroht und zahlreiche Ökosysteme gefährdet sind. ${ }^{1}$ Die sich darin offenbarende Biodiversitätskrise ${ }^{2}$ macht selbst vor den an sich streng geschützten Gebieten des Netzes Natura 2000 nicht halt. Exemplarischen Beleg bieten dafür die Ergebnisse des FFH-Berichts 2019, die erkennen lassen, dass nur etwa $30 \%$ der dort geschützten natürlichen Lebensraumtypen einen günstigen Erhaltungszustand aufweisen, während sich $32 \%$ in einem ungünstig-unzureichenden und sogar $37 \%$ in einem ungünstig-schlechten Zustand befinden. ${ }^{3}$

Auch wenn verschiedene Gefährdungsfaktoren hierfür verantwortlich sind, ist die Belastung nährstoffempfindlicher Lebensraumtypen mit Stickstoff eine der wesentlichen Ursachen. ${ }^{4}$ Der Eintrag reaktiver Stickstoffverbindungen, der durch die Landwirtschaft, durch Verbrennungspro-

1) Helmholz-Zentrum für Umweltforschung GmbH (UFZ), Das „Globale Assessment" des Weltbiodiversitätsrates IPBES. Auszüge aus dem "Summary für policymakers“, Stand: 6.5.2019.

2) Bundesamt für Naturschutz (BfN), Agrar-Report 2017. Biologische Vielfalt in der Agrarlandschaft, Juni 2017, S. 6.

3) Sutton et al., 2011, The European nitrogen assessment: sources, effects, and policy perspectives, S. 463f.; Bundesministerium für Umwelt, Naturschutz und nukleare Sicherheit (BMU), Die Lage der Natur in Deutschland. Ergebnisse von EU-Vogelschutz- und FFH-Bericht, 19.5.2020, S. 5 .

4) Vgl. nur Sachverständigenrat für Umweltfragen (SRU), Stickstoff: Lösungsstrategien für ein drängendes Umweltproblem, Sondergutachten 2015, S. 21; ders., Für einen flächenwirksamen Insektenschutz, Stellungnahme Oktober 2018, S. 22 f.; Finck/ Heinze/Raths/Riecken/Ssymank, Rote Liste der gefährdeten Biotoptypen Deutschlands, dritte fortgeschriebene Fassung 2017, Naturschutz und Biologische Vielfalt, Heft 156, 2017, S. 123. 